\title{
The Actual Cause of Gender Difference after Coronary Artery Bypass Grafting
}

\author{
Zihan Suo ${ }^{1, *}, \dagger$, Yuxuan Yang ${ }^{2, \dagger}$, and Jianing Zhang ${ }^{3, \dagger}$ \\ ${ }^{1}$ School of Life Science, University of Liverpool, L69 7ZB, Crown St., Liverpool, England \\ ${ }^{2}$ School of Life Science, University of Liverpool, L69 7ZB, Crown St., Liverpool, England \\ ${ }^{3}$ School of Statistics and Data Science, Nankai University, 300071, 94 Weijin Road, Tianjin, China \\ ${ }^{\dagger}$ These authors contributed equally.
}

\begin{abstract}
Coronary Artery Bypass Grafting (CABG) surgery is a widely practiced procedure among treatments of coronary heart disease. While being able to significantly prolong the life of patients, it is a high-risk procedure characterized by a relatively high 30-day postoperative mortality. The risk and benefits of this procedure have made it to be a focus of research in recent years. Thereinto, research was dedicated to exploring postoperative short-term mortality rate, which is affected by various factors including gender. Based on statistics, a clear pattern is demonstrated that the mortality of women is significantly greater than men. Furthermore, various confounding factors are involved, including preoperative status, physiological difference, and susceptibility for complications. By taking these factors into account, this review aims at establishing the actual cause of gender difference in short-term mortality after CABG. Besides, feasible approaches to lower the overall mortality are proposed, especially by targeting factors that led to the elevated mortality in women. These results will offer a guideline for CABG treatment.
\end{abstract}

\section{Introduction}

Coronary heart disease is a severe heart disease in which arteries are unable to deliver enough blood with oxygen to heart. It is becoming the leading cause of death in worldwide. According to the data collected from WHO in 2004, approximate 3.8 million men and 3.4 million women died from coronary heart disease each year[1]. CABG helps improve blood flow to the heart for patients who have severe coronary heart disease[2]. When medical and interventional treatments are not effective in relieving coronary heart disease, surgery seems to be reliable and effective[3]. Therefore, CABG is thought to be the best way to treat coronary heart disease. However, there are still risks of postoperative death from the infection during the surgery or complications after the surgery, though $\mathrm{CABG}$ can increase the lifespan of patients. There is a significant difference for the postoperative mortality between men and women. In this perspective, the current research of CABG, especially those talking about gender difference in postoperative mortality, are reviewed systematically. We will summarize the causation of deaths and the differences in mortality between gender. Then the current limitations/drawbacks and further improvements will be discussed. Meanwhile, some preventive measures will also be indicated to reduce the risk of postoperative death of CABG.

\section{Current research about CABG}

Since the invention of $\mathrm{CABG}$, thousands of $\mathrm{CABG}$ surgery have been performed each year[4]. Compared with other forms of treatment, $\mathrm{CABG}$ is more capable of extending the life of patients with symptomatic left main coronary artery stenosis or stenosis of the three main coronary vessels $[5,6]$. However, the effect is no longer significant after 10-12 year[7], which means the operation is only significantly efficacious in a relatively short term. Thanks to its effectiveness in saving patients with coronary vessel diseases, CABG has become one of the most studied surgeries[3], with short-term mortality being one of the most frequently discussed topics. Many of these studies have noticed several factors that result in difference in short-term mortality, which will be talked about in the following part.

\subsection{Method}

\subsubsection{Method of searching for research}

Before investigating any of the factors, it is worth mentioning the information retrieval methods for literatures. Specifically, we reviewed references published between 1980 and 2018 through the scholar search engines with the following key words: (gender

*Corresponding author: hlzsuo@liverpool.ac.uk 
OR women OR factors) AND mortality AND coronary artery bypass surgery.

\subsubsection{Method used in the papers}

It is intriguing to know how these studies collected data. Most of the studies get patient information and mortality from databases related to CABG, considering mortality is difficult to calculate directly by giving out questionnaires to patients. Most of these studies also applied randomized controlled trials, which make the studies more convincing.

We also found some statistical techniques applied in those papers, which would not be covered in the following discussion. Some studies used $\chi$-squared test of homogeneity to test whether mortality was different between different groups. That is reasonable because the exact test evaluates the observed differences between the sets arose by chance. For the model to be adjusted for different factors, some other studies used logistic regression, which is usually used to model the possibility of a certain event. Mantel-Haenszel $\chi$-squared test was also used to process data from different years.

\subsection{Decrease of short-term mortality after CABG by year}

Many studies have noticed that in-hospital mortality has decreased significantly over the years[8,9]. One study carried out in British Columbia shows that overall 30-day mortality has decreased significantly, from $3.1 \%$ to $1.9 \%$ in the 14-year scale[8]. Another New York study, which was carried out earlier, find that in-hospital mortality has dropped from $3.52 \%$ to $2.78 \%$ in 4 years. Few studies talk about why mortality has dropped over the years, which might be ascribed to advances in surgical techniques and experience.

\subsection{Ethnic and regional difference in short-term mortality after CABG}

There are few research talking about whether ethnic and region are factors affecting short-term mortality after CABG. Based on hospital administrative data in British Columbia, Canada, one paper looked into the difference between patients of different races after CABG[10]. It used a validated surname algorithm to identify the ethnicity of patients, which was a mix of Nam Pehchan computer program and Chinese surname list. Another paper directly compared mortality of patients having CABG in urban hospitals in China and US[11], which could be viewed as a comparison of different regions.

In general, both papers stated Chinese have a higher in-hospital mortality. To be specific, in the 2007 to 2008 and 2010 period, the in-hospital mortality for patients aged $>65$ years old was significantly higher in China. The odds ratio was 1.43, 1.79, and 1.73 from the unadjusted, age- and sex-adjusted, and age-, sex-, comorbidities-adjusted, respectively[11]. In the other research, the odds ratio was $2.36,1.63,1.45$ for Chinese vs. White, South Asians vs. White and Chinese vs. South
Asians[10]. The researchers also admitted that the results need other corroboration.

\subsection{Gender difference in short-term mortality after CABG}

Various studies have proved gender difference in unadjusted early mortality after CABG[4, 8]. In contrast, late mortality showed no significant difference between men and women. The difference in short term mortality can be accounted for multiple factors, e.g., comorbidities, age, body habitus, etc. While in some of the studies, the difference is still significant even adjusted for multiple factors[12], in other papers it does not. To be noted, younger women $(<50$ years old) have a higher 30 -day, in-hospital and post-dis-charge mortality than men[4].

In the following part of this review, we will mainly focus on gender difference in the short-term mortality.

\section{Postoperative mortality}

\subsection{Causation of postoperative deaths}

As a revascularization procedure that significantly improves prognosis in stable coronary artery disease, the mechanism of CABG is complicated. Therefore, it is still considered a high-risk procedure even with improvements in techniques[13]. According to Ref. [14], about $16.5 \%$ of the patients undergone CABG was readmitted into the hospital within 30 days in New York. Thereinto, the most common reason for hospital readmission is directly operation-related, accounting for approximately $87 \%$ of the patients. In this case, the high risk involved in the operation procedure might be a major contributor to short-term postoperative mortality.

Besides the risk in the operation itself, patients may suffer from a wide range of complications after the operation, resulting in approximately $14 \%$ of the patients being readmitted into the hospital within 30 days[15]. The most accounted complication is post-operative infections, approximately $16 \%$ of the complications can be categorised into post-operative infections (Table 1 and Figure 1)[14]. The type of infections are diverse, and many organs can be affected[15]. Among all kinds of infections, the most devastating one is deep sternal wound infection (DSWI). Although it is not the most common post-operative infection among CABG patients, its mortality rate is strikingly high, which is reported to be around $15 \%[16]$. It is much higher than the general mortality rate of the CABG patients, which is reported to be around $2 \%[17]$. This shows that DSWI is a potential risk factor for CABG postoperative mortality. Another postoperative infection that worth noticing is pneumonia. Among patients that require mechanical ventilation for more than two days, the prevalence of pneumonia can reach $35.2 \%[18]$, and 30-day mortality in high-risk patients can reach $42 \%[19]$. Pneumonia is usually caused by a wide spectrum of bacteria, i.e., increased the difficulty in choosing an appropriate antibiotic therapy, which often leads to a poor prognosis[18]. 
Table 1: Most common complications among readmissions

\begin{tabular}{cc}
\multicolumn{2}{c}{ after } \\
\hline Postoperative complication & \% \\
\hline Postoperative infections & 16.9 \\
\hline Heart failure & 12.8 \\
\hline Cardiac dysrhythmias & 6.3 \\
\hline Chest pain & 4.7 \\
\hline Pneumonia & 4.0 \\
\hline Myocardial fraction & 2.6 \\
\hline Others & 52.7
\end{tabular}

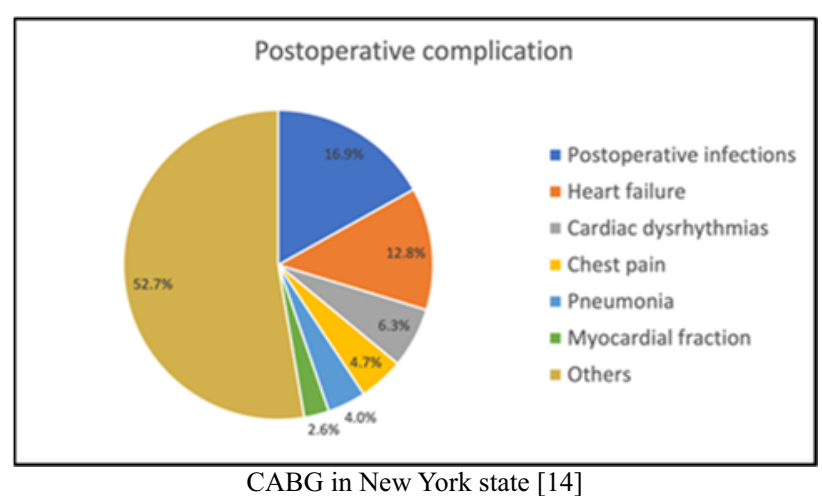

Figure 1: Proportion of most common complications among readmissions after CABG in New York state [14]

Congestive heart failure is another major category of well-observed postoperative complication, accounting for around $10 \%$ of all recorded complications[20]. The cause of congestive heart failure can be divided into two categories. The first category of causation is directly CABG operation-related, represented by myocardial ischemia. Around half of the patient will experience postoperative myocardial ischemia, but this is usually asymptomatic[21]. Furthermore, more severe case of graft failure may result in myocardial infarction, which can be observed in $5 \%$ to $10 \%$ of the patients, increasing their mortality rate to around $15 \%[22]$

\subsection{Gender difference in postoperative mortality}

Gender is a major confounding factor for both the 30-day mortality and 30-day hospital readmission risk[8, 14]. Therefore, it is vitally important to isolate the underlying cause for the difference between the two genders. Generally, at the time of hospital admission, women tend to be older and usually present in a more urgent situation, suffering from more severe comorbidities including hypertension, diabetes, cerebrovascular disease and congestive heart failure[23, 24]. These preoperative profiles may have played a role in elevating the short-term mortality rate in women. Whereas, women were also observed to have less complicated disease pattern, which requires less revascularization that decreases the mortality risk and leads to a better prognosis[25].

Intraoperative procedures may also be contributed to the higher short-term mortality in women. The physiological difference between men and women (smaller vessel size in women) may lead to prolonged bypass operation procedure and require more complicated techniques (e.g., intra-aortic balloon pump).
These factors significantly increased the mortality rate among women[26]. Studies have also found that with the technical improvements in coronary anastomosis, there is a steeper drop in mortality of women compared to men. Possible reason is that the improvement benefits more for individuals with smaller vessel size[8], providing further evidence that physiological difference is a major risk factor that increased short-term mortality in women.

Gender difference can also be observed in postoperative complications. Intriguingly, women are observed to have a decreased risk in developing DSWI[24], while another study argues that wound infections are more prevalent in women[25]. Unlike wound infections, pneumonia is observed to be not associated with gender[18]. Additionally, there is also no indication that postoperative congestive heart failure is gender related.

\section{Discussions}

\subsection{Confounding variables in the study}

Based on above literatures, the possible risk factors that women have a higher mortality rate than men after undergoing CABG have been determined. Nevertheless, according to Humphries et al., differences between men's and women's socioeconomic status and psychosocial profile may be confounding variables which affect mortality between genders [8]. Socioeconomic status (SES) is used to describe the status of a person in society. In order to measure an individual's SES, levels of education, income and professional status are used as criteria for SES evaluation [27].

\subsection{Some common complications after CABG}

As patients complete the CABG surgery, patients probably suffer negative effects from some complications. Knipp et al. claimed that within a period of time after the surgery, approximately $51 \%$ of patients developed new brain injury due to cerebral ischemia [28]. Meanwhile, the CABG may lead to cognitive decline and memory impairment. In addition, cognitive decline can be recovered in a short time, but memory impairment may last for several months [29]. However, it is worth noting that the brain lesions are not likely to be the cause of cognitive decline and memory impairment [29]. Other complications in the nervous system, for instance, stroke and some ophthalmologic abnormalities will occur after the CABG with the probability of 5\%-6\% and $13 \%-29 \%$, respectively [3]. In addition to complications in the nervous system, adverse cardiac outcomes appear to account for the significant mortality after CABG [30]. Myocardial ischemia, which is an important predictor of adverse cardiac outcomes, occurs after the surgery more prevalently than pre-operation [30]. The adverse cardiac outcomes cause cardiac morbidity and mortality was considered as significant problems after undergoing CABG [30]. 


\subsection{Prevent from the death after CABG}

Although there is a risk of death after CABG, this risk can be avoided. Some bad habits after the surgery may increase the risk of postoperative death. For example, smoking is a significant predictor of higher risk of death. According to van Domburg et al., smokers have greater probability of postoperative death because the repeat revascularization procedure will be undergone frequently [31]. Therefore, smoking cessation is highly recommended to the smoker patients who will undergo the CABG surgery or those who have already undergone the CABG surgery.

After the CABG surgery, if patients are required to undergo other noncardiac surgery, it is essential to perform a cardiac screening during the preoperative consultation. If they satisfy the ejection fraction less than $45 \%$ and right ventricular systolic pressure more than 40 $\mathrm{mmHg}$ before the noncardiac surgery, there are more likely to result in adverse cardiac outcomes after the noncardiac surgery [32]. Thus, the subsequent surgery should be rescheduled or even abandoned to ensure life safety.

\section{Conclusion}

In summary, based on literature analysis, plenty of underlying factors (e.g., preoperative status, perioperative risks, and postoperative complications) contribute to the difference in short term mortality between the two genders. It is very likely that gender per se is not a risk factor in the CABG postoperative short-term mortality. Realizing this would be helpful in future research when it comes to lowering the mortality rate, especially in lowering the elevated mortality in women. However, the confounding factors in this topic is complicated, more work may still be required to explore all possible factors that may affect mortality rates.

\section{REFERENCE}

1. Mackay, J. and G.A. Mensah, The atlas of heart disease and stroke. 2004: World Health Organization.

2. Coronary Artery Bypass Grafting (CABG). [Accessed: April 17 2021]; Available from: https://cardiacsurgery.ucsf.edu/conditions--procedur es/coronary-artery-bypass-grafting-(cabg).aspx.

3. Hawkes, A.L., et al., Outcomes of coronary artery bypass graft surgery. Vasc Health Risk Manag, 2006. 2(4): p. 477-84.

4. Vaccarino, V., et al., Sex differences in hospital mortality after coronary artery bypass surgery: evidence for a higher mortality in younger women. 2002. 105(10): p. 1176-1181.

5. Grover, F.L., K.E. Hammermeister, and C.J.T.A.o.t.s. Burchfiel, Initial report of the Veterans Administration preoperative risk assessment study for cardiac surgery. 1990. 50(1): p. 12-26.
6. Lancet, E.C.S.S.G.J.T., Prospective Randomised Study of Coronary Artery Bypass Surgery In Stable Angina Pectoris: Second Interim Report by the. 1980. 316(8193): p. 491-495.

7. Cundiff, D.K.J.M.M.g.m., Coronary artery bypass grafting $(\mathrm{CABG})$ : reassessing efficacy, safety, and cost. 2002. 4(2): p. 7-7.

8. Humphries, K.H., et al., Significant improvement in short-term mortality in women undergoing coronary artery bypass surgery (1991 to 2004). 2007. 49(14): p. 1552-1558.

9. Hannan, E.L., et al., Improving the outcomes of coronary artery bypass surgery in New York State. 1994. 271(10): p. 761-766.

10. Gasevic, D., et al., Outcomes following percutaneous coronary intervention and coronary artery bypass grafting surgery in Chinese, South Asian and White patients with acute myocardial infarction: administrative data analysis. 2013. 13(1): p. 1-7.

11. Zheng, Z., et al., Comparing outcomes of coronary artery bypass grafting among large teaching and urban hospitals in China and the United States. 2017. 10(6): p. e003327.

12. Kim, C., et al., A systematic review of gender differences in mortality after coronary artery bypass graft surgery and percutaneous coronary interventions. 2007. 30(10): p. 491-495.

13. Lee, C.W., et al., Coronary Artery Bypass Surgery Versus Drug-Eluting Stent Implantation for Left Main or Multivessel Coronary Artery Disease. 2016. 9(24): p. 2481-2489.

14. Hannan, E.L., et al., 30-Day Readmissions After Coronary Artery Bypass Graft Surgery in New York State. JACC: Cardiovascular Interventions, 2011. 4(5): p. 569-576.

15. Montrief, T., A. Koyfman, and B. Long, Coronary artery bypass graft surgery complications: A review for emergency clinicians. American Journal of Emergency Medicine, 2018. 36(12): p. 2289-2297.

16. Filsoufi, F., et al., Epidemiology of deep sternal wound infection in cardiac surgery Journal of cardiothoracic and vascular anesthesia, 2009. 23(4): p. 488-494.

17. Doenst, T., et al., PCI and CABG for Treating Stable Coronary Artery Disease. Journal of the American College of Cardiology, 2018. 73(8): p. 964-976.

18. He, S., et al., Ventilator-associated pneumonia after cardiac surgery: A meta-analysis and systematic review. Journal of Thoracic and Cardiovascular Surgery, 2014. 148(6): p. 3148-3155.

19. Allou, N., et al., Postoperative pneumonia following cardiac surgery in non-ventilated patients versus mechanically ventilated patients: is there any difference? Critical Care, 2015. 19(116).

20. Fox, J.P., et al., Hospital-Based, Acute Care Use Among Patients Within 30 Days of Discharge After Coronary Artery Bypass Surgery. Ann Thorac Surg, 
2013. 96: p. 96-104.

21. Smith, R.C., J.M. Leung, and D.T. Mangano, Postoperative myocardial ischemia in patients undergoing coronary artery bypass graft surgery. S.P.I. Research Group Anesthesiology, 1991. 74(3): p. 464-473.

22. Laflamme, M., et al., Management of early postoperative coronary artery bypass graft failure. Interact Cardiovasc Thorac Surg, 2012. 14(4): p. 452-456.

23. Filardo, G., et al., Excess short-term mortality in women after isolated coronary artery bypass graft surgery. Open Heart, 2016. 2016(3): p. 1-6.

24. Saxena, A., et al., Sex differences in outcomes following isolated coronary artery bypass graft surgery in Australian patients: analysis of the Australasian Society of Cardiac and Thoracic Surgeons cardiac surgery database. European Journal of Cardio-Thoracic Surgery, 2012. 41: p. 755-762.

25. Al-Alao, B.S., et al., Gender influence in isolated coronary artery bypass graft surgery: a propensity match score analysis of early outcomes. Gen Thorac Cardiovasc Surg, 2012. 60(7): p. 417-424.

26. Sharoni, E., et al., Is Gender an Independent Risk Factor for Coronary Bypass Grafting? Thorac Cardiovasc Surg, 2009. 57(4): p. 204-208.

27. Yang, F.M. and S.G. Leveille, Chapter 95 Morbidity, Disability, and Mortality, in Women and Health (Second Edition), M.B. Goldman, R. Troisi, and K.M. Rexrode, Editors. 2013, Academic Press. p. 1401-1413.

28. Knipp, S.C., et al., Cognitive outcomes three years after coronary artery bypass surgery: relation to diffusion-weighted magnetic resonance imaging. Ann Thorac Surg, 2008. 85(3): p. 872-9.

29. Knipp, S.C., et al., Evaluation of brain injury after coronary artery bypass grafting. A prospective study using neuropsychological assessment and diffusion-weighted magnetic resonance imaging. Eur J Cardiothorac Surg, 2004. 25(5): p. 791-800.

30. Smith, R.C., et al., Postoperative myocardial ischemia in patients undergoing coronary artery bypass graft surgery. The Journal of the American Society of Anesthesiologists, 1991. 74(3): p. 464-473.

31. van Domburg, R.T., et al., Smoking cessation reduces mortality after coronary artery bypass surgery: a 20-year follow-up study. Journal of the American College of Cardiology, 2000. 36(3): p. 878-883.

32. Mookadam, F., et al., Risk of adverse events after coronary artery bypass graft and subsequent noncardiac surgery. Future Cardiol, 2011. 7(1): p. 69-75. 